\title{
Serine Proteases Profiles of Leishmania (Viannia) Braziliensis Clinical Isolates With Distinct Susceptibilities to Antimony
}

\section{Anabel Zabala-Peñafiel}

Fiocruz

Geovane Dias-Lopes

Fiocruz

\section{Léa Cysne-Finkelstein}

FIOCRUZ: Fundacao Oswaldo Cruz

\section{Fátima Conceição-Silva}

Fundacao Oswaldo Cruz

\section{Luciana de Freitas Campos Miranda}

Fundacao Oswaldo Cruz

\section{Aline Fagundes}

Fundacao Oswaldo Cruz

Armando de Oliveira Schubach

Fundação Oswaldo Cruz

Maria Ines Fernandes Pimentel

Fundacao Oswaldo Cruz

Franklin Souza-Silva

Fundacao Oswaldo Cruz

Lucas de Almeida Machado

Fundacao Oswaldo Cruz

Carlos Roberto Alves ( $\nabla$ calves@ioc.fiocruz.br)

Laboratório de Biologia Molecular e Doenças Endêmicas, Instituto Oswaldo Cruz, Fundação Oswaldo Cruz, Rio de Janeiro, Brasil. https://orcid.org/0000-0001-8703-426X

\section{Research}

Keywords: American Tegumentary Leishmaniasis, serine proteases, subtilisins, tryparedoxin peroxidase, clinical isolates, susceptibility, Glucantime ${ }^{\circledR}$

Posted Date: January 20th, 2021

DOl: https://doi.org/10.21203/rs.3.rs-79982/v2 
License: (c) (i) This work is licensed under a Creative Commons Attribution 4.0 International License. Read Full License

Version of Record: A version of this preprint was published at Scientific Reports on July 9th, 2021. See the published version at https://doi.org/10.1038/s41598-021-93665-z. 


\section{Abstract}

Glucantime ${ }^{\circledR}\left(\mathrm{Sb}^{\vee}\right)$ is the first-line treatment against leishmaniasis in South America. Its effectiveness has been associated with modulation of the parasite detoxification system that, in turn, is related to serine proteases such as subtilisins. In this study, 12 Leishmania (Viannia) braziliensis isolates from patients that presented clinical cure (Responders - R) and relapse or therapeutic failure (Non-responders $\mathrm{NR}$ ) were used. The parasites were assessed by in vitro susceptibility to $\mathrm{Sb}^{\mathrm{III}}$ and $\mathrm{Sb}^{\mathrm{V}}$, serine proteases activity - measured with z-FR-AMC as substrate and specific inhibitors - and expression of subtilisins and tryparedoxin-peroxidase (TXNPx). In vitro susceptibility of axenic amastigotes to Sb ${ }^{\text {III }}$ showed a significant difference between $R$ and NR groups. TLCK inhibited almost $100 \%$ of activity in both axenic amastigotes and promastigotes while AEBSF inhibited around $70 \%$, and PMSF showed lower inhibition of specific isolates. Principal component and clustering analysis yielded one homogeneous cluster with only NR isolates and three heterogeneous clusters with R and NR isolates. Additionally, transcripts of subtilisins (LbrM.13.0860 and LbrM.28.2570) and TXNPx (LbrM.15.1080) were detected in promastigotes and axenic amastigotes from both groups. The data presented here show a phenotypic heterogeneity among the parasites, suggesting that exploration of in vitro phenotypes based on $\mathrm{Sb}^{\mathrm{III}}$ and serine proteases profiles can aid in the characterization of $L$. (V.) braziliensis clinical isolates.

\section{Introduction}

Leishmaniasis is a neglected tropical disease that affects around 1 million people worldwide, causing 26 000 to 65000 deaths per year (1). Leishmania spp. are eukaryotic protozoan parasites responsible for a broad range of clinical manifestations, such as cutaneous, mucocutaneous and visceral forms, which depend on the parasite species and immune state of the mammalian host (2). In the American continent, Leishmania mexicana complex and subgenus Viannia species cause cutaneous and mucocutaneous forms that can develop into localized, disseminated or diffuse lesions, which is known as American Tegumentary leishmaniasis (ATL) (1).

A pentavalent antimony formulation - Glucantime ${ }^{\circledR}\left(\mathrm{Sb}^{\vee}\right)$ - is the first-line treatment against $\mathrm{ATL}$ and has not changed in the last 70 years (1). In South America, this treatment doses (low and high dose) vary according to therapeutic response patterns seen in specific geographical areas (3-5). Its mechanism of action is still not completely understood, but, it has been well accepted the hypothesis that $\mathrm{Sb}^{\mathrm{V}}$ needs to be reduced to its trivalent form (Sb"II), either directly by the parasite or within the host macrophages, to excel leishmanicidal activity (6-8).

Moreover, the most abundant Leishmania spp. thiol, trypanothione reductase $\left[\mathrm{T}(\mathrm{SH})_{2}\right]$, is part of the parasite trypanothione reductase system which is a unique detoxification defence mechanism that relies on four key enzymes: trypanothione synthetase (TryS), trypanothione reductase (TR) and tryparedoxin (TXN) and tryparedoxin peroxidase (TXNPx) (9). This system is involved in the detoxification of metal ions, including reduction of $\mathrm{Sb}^{\mathrm{V}}$ to $\mathrm{Sb}^{\mathrm{III}}$, within the macrophage phagolysosome $(6,7)$. It is also 
hypothesised that $\mathrm{Sb}^{\mathrm{V}}$ reduction might happen through the oxidation of $\mathrm{T}(\mathrm{SH})_{2}$ forming a stable complex - SbllI $(T S)_{2}$ - that could be pumped out of the cells through parasite membrane transporters $(6,9)$. Interestingly, parasite's resistance towards $\mathrm{Sb}^{\mathrm{V}}$ has been associated with increased trypanothione levels and a decreased capacity to reduce $\mathrm{Sb}^{\mathrm{V}}(10-12)$. Indeed, increased abundance of TXNPx has been correlated with an enhanced thiol redox potential in $\mathrm{Sb}^{\mathrm{V}}$ resistant parasites, not only in lab-generated strains $(13,14)$ but also in clinical isolates $(15)$.

Furthermore, some studies have shown that parasite enzymes are involved in the reduction of $\mathrm{Sb}^{\mathrm{V}}$ to $\mathrm{Sb}^{\mathrm{III}}$ and Leishmania spp. resistance towards $\mathrm{Sb}^{\mathrm{V}}(10,16,17)$. Particularly, serine proteases have been extensively studied in American Leishmania spp. due to their functional interrelationship in parasite physiology and their potential as therapeutic targets (18-24). Experimental evidence has demonstrated that these enzymes can act as signal peptidases, maturases of other proteins, and can have a metacaspase-like activity $(20,25)$. Among them, subtilisins are proposed to have a functional interrelationship with other Leishmania spp. proteins, suggesting key functions of these enzymes in parasite physiology such as modulation of the trypanothione reductase system through direct action over $\operatorname{TXNPx}(18,25)$.

In this context, subtilisins are correlated to the balance of cytosolic and mitochondrial TXNPx levels in $L$. (L.) donovani clinical isolates. Subtilisin knockout promastigotes of these parasites failed to differentiate into viable amastigotes and their TXNPx peptide abundance was decreased, which suggested that subtilisins can act as maturases of specific proteins or pathways (25). Although, in L. (V.) braziliensis, subtilisins have distinct subcellular distributions and expression (26) it has not been assessed yet if these enzymes excel a similar role during parasite differentiation; and if impairment of trypanothione reductase system has any relation with $\mathrm{Sb}^{\vee}$ resistant phenotypes.

This study aims to contribute in the understanding of Glucantime ${ }^{\circledR}$ susceptibility based on $L$. $(V$.) braziliensis clinical isolates. Gathered data here incorporate new information on the heterogenic profile of clinical isolates, assessing their in vitro susceptibility towards $\mathrm{Sb}^{\mathrm{V}}$ and $\mathrm{Sb}^{\mathrm{III}}$ as a direct relation to serine proteases of this parasite. Additionally, transcripts of some enzymes of the parasite detoxification system as subtilisins (LbrM.13.0860 and LbrM.28.2570) and TXNPx (LbrM.15.1080) were accessed.

\section{Results}

\section{In vitro susceptibility to antimony}

Both, $\mathrm{Sb}^{\mathrm{III}}$ and $\mathrm{Sb}^{\mathrm{V}}$, were unable to discriminate susceptibility profiles between promastigote forms of responders $(R)$ and non-responders (NR) isolates. The mean $I_{50}$ value of $R$ versus NR group was very close which did not allow discrimination among them (Fig. 1A and 1B). Meanwhile, the results showed a significant difference between the axenic amastigotes grouped as NR and R exposed to $\mathrm{Sb}^{\mathrm{II}}(p<0.05$, 
Fig. 1C). Conversely, these forms did not respond well to $\mathrm{Sb}^{\vee}$ exposure and we were not able to discriminate among the groups (Fig. 1D). The $\mathrm{IC}_{50}$ values are summarised in Supplementary file 1.

$\mathrm{Sb}^{\mathrm{V}}$ was toxic for only two isolates, $\mathrm{R} 1(0.005 \pm 0.27 \mathrm{mg} / \mathrm{mL})$ and $\mathrm{R} 5(0.056 \pm 0.14 \mathrm{mg} / \mathrm{mL})$ while the $I_{50}$ values of all other isolates were $\geq 0.295 \mathrm{mg} / \mathrm{mL}$ (Supplementary file 1). Regarding the axenic amastigotes, $\mathrm{Sb}^{\mathrm{V}}$ did not differ among the isolates since the values were very similar, for example, R1 $(0.007 \pm 0.16 \mathrm{mg} / \mathrm{mL})$ while NR3 $(0.001 \pm 0.4 \mathrm{mg} / \mathrm{mL})$. However, Sb ${ }^{\text {III }}$ was selectively more toxic for axenic amastigotes from the R group: R3 $\left(9 \times 10^{-7} \pm 0.39 \mathrm{mg} / \mathrm{mL}\right)$ and $R 5(0.0003 \pm 0.24 \mathrm{mg} / \mathrm{mL})$.

\section{Quantification of protease activity}

All analysed samples presented protease activity, as they were able to hydrolyse the Z-FR-AMC substrate, a chromogenic synthetic peptide appropriate for serine proteases. Promastigotes had a fairly similar activity without inhibition (W/i) between promastigotes ( $76 \pm 0.07$ to $1025 \pm 0.07 \mu \mathrm{mol}$. $\mathrm{min}^{-}$ ${ }^{1} \mathrm{mg}$ of protein ${ }^{-1}$ ) and axenic amastigotes (133 \pm 0.003 to $1063 \pm 1.2 \mu \mathrm{mol} . \mathrm{min}^{-1} \mathrm{mg}$ of protein ${ }^{-1}$ ) (Fig. 2 and Supplementary file 2). The specificity of serine proteases activity was assessed by measuring the hydrolysis of the Z-FR-AMC substrate in the presence of selective inhibitors (Fig. 2 and Supplementary file 2). In general, enzymatic activities in presence of TLCK were significantly lower compared to the other serine proteases inhibitors. For promastigotes, the residual activities ranged from $9 \pm 0.03$ to $241 \pm 0.1 \mu \mathrm{mol}$. $\min ^{-1} \mathrm{mg}$ of protein ${ }^{-1}$, while for axenic amastigotes from $8 \pm 0.04$ to $161 \pm 0.09 \mu \mathrm{mol}$. $\mathrm{min}^{-1} \mathrm{mg}$ of protein ${ }^{-1}$. Additionally, AEBSF in promastigotes ranged from $40 \pm 0.03$ to $937 \pm 0.09 \mu \mathrm{mol}$. $\mathrm{min}^{-1} \mathrm{mg}$ of protein ${ }^{-1}$, and in axenic amastigotes ranged from $14 \pm 0.04$ to $766 \pm 0.1 \mu \mathrm{mol} . \mathrm{min}^{-1} \mathrm{mg}$ of protein ${ }^{-1}$. Conversely, PMSF residual activities were the highest among all isolates (Fig. 2).

Furthermore, inhibition assays using E-64, a cysteine protease inhibitor, suggests that, under our controlled conditions, Z-FR-AMC was not cleaved by cysteine proteases from the isolates' protein extracts (Fig. 2). The majority of residual activities were similar or even higher than those W/i. Exceptionally, only the axenic amastigote extracts from NR1 $\left(147 \pm 0.1 \mu \mathrm{mol}^{.} \mathrm{min}^{-1} \mathrm{mg}\right.$ of protein $\left.{ }^{-1}\right)$, NR5 $\left(250 \pm 0.5 \mu \mathrm{mol} . \mathrm{min}^{-1} \mathrm{mg}\right.$ of protein $\left.{ }^{-1}\right)$ and NR7 $\left(899 \pm 0.2 \mu \mathrm{mol} . \mathrm{min}^{-1} \mathrm{mg}\right.$ of protein $\left.{ }^{-1}\right)$ showed a significant difference when compared to their respective $\mathrm{W} / \mathrm{i}$ controls.

The inhibition percentage of each serine inhibitor was calculated using each isolate $\mathrm{W} / \mathrm{i}$ as a comparison (Supplementary file 3). TLCK showed the strongest inhibition with almost $100 \%$ of inhibition for R1, R2, R3, R4, NR1 and NR2 both in promastigotes and axenic amastigotes extracts. Similarly, AEBSF strongly inhibited the same group of isolates $(70 \%)$ but only for the promastigotes' extracts, while for the axenic amastigotes extracts the inhibition was dispersed, only R1, R3 and NR6 were $70 \%$ inhibited. On the contrary, PMSF showed the lowest inhibition rates. In promastigotes extracts of R3, R4, NR6 and NR7, and axenic amastigotes extracts of R3, R5, NR3, NR6 and NR7 it inhibited less than $35 \%$. Altogether these 
results indicated that these isolates have a distinct quantitative profile for serine protease activities in both assayed parasite forms.

\section{Cluster analysis}

The first three PCs explained approximately $80 \%$ of the data variance. Based on our analysis of the total sum of squares as a function of the number of clusters (Supplementary file 4) we opted for using five clusters: Cluster 1, Cluster 2, Cluster 3, Cluster 4, and Cluster 5 (Fig. 3). There was one cluster that contained, exceptionally, only one clinical isolate (Cluster 3: NR3). One homogenous cluster containing two isolates from the same clinical group (Cluster 2: NR1, NR4) while the remaining three were heterogeneous (Cluster 1: NR6, R5; Cluster 4: R1, R3, NR5, NR7; and Cluster 5: NR2, R2, R4).

Interestingly, in the heterogeneous Cluster 1 , the distances between both members for all variables ranged from 0.2 to 0.7 , except for TLCK inhibition over promastigotes (0.04) and axenic amastigotes (0.9) (Supplementary file 5). Moreover, within Cluster 4, another heterogeneous cluster with two R and two NR isolates, TLCK inhibition between R and NR was the highest for both promastigotes and axenic amastigotes, while, PMSF and AEBSF values ranged from 0.06 to 0.85 without an established pattern (Supplementary file 6). Also, in Cluster 5, a cluster containing two R and one NR isolate, there was a pattern among variables with all distance values lower than 0.56, exceptionally, TLCK inhibition of NR3 versus R6 axenic amastigotes being the highest distance (0.72) (Supplementary file 7). Regarding Sb ${ }^{\text {III }}$ distance within Cluster 4 and Cluster 5 , there is no value higher than 0.43 .

\section{Gene transcripts}

Subtilisins (LbrM.13.0860 and LbrM.28.2570) and tryparedoxin peroxidase (LbrM.15.1080) genes were investigated in both parasite biological forms of the R and NR group, by detection of RNA-transcripts of 200bp, 162bp, and 166bp, respectively (Fig. 4). In general, these RNA-transcripts were detected in all the cDNA samples, but with distinct bands intensity according to the biological form of the parasite, when compared to the profile of the transcripts of the 40S Ribosomal Protein S8 housekeeping gene LbrM.24.2160 which remained constant for all isolates (Fig. 4D). Our data showed that LbrM.13.0860 gene transcripts (Fig. 4A) were not as well detected as LbrM.28.2570 and LbrM.15.1080 gene transcripts (Fig. 4B and 4C), both in promastigotes and axenic amastigotes of all isolates.

\section{Discussion}

The hypothesis that $L$. $(V$.) braziliensis clinical isolates have, indeed, different response profiles towards antimony was corroborated in this study by in vitro susceptibility experiments using $\mathrm{Sb}^{\mathrm{V}}$ and $\mathrm{Sb}^{\mathrm{III}}$. It is known that antimonial therapy failure and resistance do not only depend on host characteristics (nutrition, immune status, comorbidities, inadequate drug doses and treatment follow-up) but also on parasite factors (strains innate susceptibility, virulence factors, biologic profile) (12-15,27-29). In this context, Leishmania spp. capacity to alternate between clonal and sexual reproduction increases their 
diversity, plasticity and biological capacity to expand under different stress conditions (30). Exposure and resistance towards several drugs could be a developed capacity to adapt and survive within hosts.

The $\mathrm{IC}_{50}$ values obtained in this study showed that $L$. $(V$.) braziliensis clinical isolates were better distinguished due to the $\mathrm{Sb}^{\mathrm{III}} \mathrm{IC}_{50}$ values over the axenic amastigote forms. Promastigotes showed a 1.9fold difference while axenic amastigotes presented a significant 3.1-fold difference between the R and NR group. However, R5 and NR2 promastigotes did not follow this observation, since they had almost the same $\mathrm{IC}_{50}$ value. $\mathrm{Sb}^{\mathrm{V}}$ was toxic for promastigotes of each clinical isolate and showed a 2-fold difference between the $\mathrm{R}$ and NR group. These results are in concordance with another study using $L$. $(V$.) braziliensis promastigotes, isolated from clinical samples of the same endemic area, that showed $\mathrm{Sb}^{\mathrm{V}}$ $\mathrm{IC}_{50}$ minimum and maximum values of $0.37 \pm 0.09$ and $5.75 \pm 0.26 \mathrm{mg} / \mathrm{mL}$ (13). Similarly, another study showed great variance among $\mathrm{Sb}^{\vee} \mathrm{IC}_{50}$ values, with a 3-fold difference between promastigotes from poor/bad and cured/good clinical response to antimonial therapy (31). It has been observed that $L$. $(V$.) braziliensis isolates, circulating in the state of Rio de Janeiro, share common genetic traits but have different responses to Glucantime ${ }^{\circledR}$ treatment (32). The varied in vitro susceptibility showed in this study reinforces the phenotypical heterogeneity reported for this species.

Even though intracellular amastigote is the "gold standard" model to test susceptibility, axenic amastigotes have been previously used to evaluate response towards $\mathrm{Sb}^{\mathrm{V}}$, which was found to be stage and strain-specific in $L$. (L.) donovani (33) and $L$. (L.) infantum (34). Furthermore, it is important to remember that assays with intracellular amastigotes are difficult to standardize and strongly depend on the type of host cell and the medium used $(15,35)$, which might bias the final results. Therefore, axenic amastigotes are a feasible model to characterize in vitro phenotypes since they maintain similar morphology, metabolic and virulence genes expression profiles as intracellular amastigotes (36-40)

The fact that promastigotes and axenic amastigotes have different in vitro susceptibilities, led us to evaluate the possible correlation of these phenotypes with virulence factors - serine proteases - under a biochemical approach. The serine protease activity measured by Z-FR-AMC, a substrate for serine proteases such as cathepsins, kallikrein and plasmin (41), was relatively the same among promastigotes and axenic amastigotes. Serine enzymatic activity is generally confirmed by using PMSF, AEBSF and TLCK inhibitors (26). Besides, PMSF and AEBSF are known to inhibit a broad range of serine proteases including subtilisins while TLCK has a greater preference for Leishmania spp. oligopeptidases $(20,42-$ 44). The inhibition profiles seen in this study were different depending on each inhibitor assayed, suggesting that there are different groups or isoforms of serine proteases as part of the protease-network of each clinical isolate, and its respective biological forms.

Furthermore, cluster analysis was performed to investigate if the in vitro phenotypes correlate with the clinical response of each clinical isolate. Cluster 3 contained only one isolate, NR3, which was closely similar to the isolates from homogeneous Cluster 2 (NR1, NR4). Cluster analysis shows the associations 
between in vitro susceptibility and clinical response. However, additional studies with more isolates from other geographical areas are necessary to explore and reinforce the associations found here.

On the other hand, the presence of heterogeneous clusters was better understood once we compared the pairwise distance between the members of each cluster. This analysis showed a similar pattern of PMSF and AEBSF inhibition over both parasite biological forms while TLCK inhibition was significantly more varied among the heterogeneous clusters. This may suggest that independently of each isolate clinical response, they have common enzymatic and in vitro Sb"I susceptibility traits. This observation supports the hypothesis that parasites of the subgenus Viannia are a polyclonal population with high genetic variability and, consequently, phenotypic diversity $(37,45)$. Other studies have shown that genetic and phenotypic characteristics among different $L$. (V.) braziliensis strains are associated with different clinical manifestations and drug resistance $(32,46)$. Additionally, the variation among serine proteases inhibition indicates specific traits among the conformation of their serine protease network since the specificity of PMSF, AEBSF and TLCK is different $(42,47)$. Therefore, $L$. (V.) braziliensis isolates can be composed of distinct enzymatic profiles that influence host-parasite interactions and, consequently, the success or failure of specific drugs.

Additionally, based on the previous role seen for subtilisins as maturases of TXNPx $(18,26)$, our study examined the expression of subtilisin transcripts from SB clan and S8 family (LbrM.13.0860 and LbrM.28.2570) and TXNPx (LbrM.15.1080). In general, almost all isolates (promastigotes and axenic amastigotes) expressed the three mentioned genes. This finding suggests that subtilisins and TXNPx are expressed by $L$. (V.) braziliensis clinical isolates, but its level of expression varies depending on the isolate phenotype. Interestingly, a proteomic study using lab-generated $L$. (V.) braziliensis NR/resistant strains showed that TXNPx abundance was significantly higher than R/susceptible strains (48). Additionally, another study using $L$. (L.) donovani clinical isolates correlated TXNPx higher amplification levels with antimony resistance phenotypes (49). However, is important to bear in mind that this study did conventional PCR which is a qualitative method, thus, QPCR and proteomics experiments, which are more sensitive quantitative methods, need to be performed to quantify these transcripts expression and explore their relationship with different in vitro phenotypes.

Leishmania spp. resistance towards antimony treatment is an increasing multifactorial phenomenon, and several virulence factors have been studied and related to resistant phenotypes in clinical isolates $(15,50-52)$. The present study adds to this discussion by bringing proteases to focus, one of the most studied parasite virulence factors in the last 30 years $(18,21,53)$. The data presented here show the possibility to use serine proteases to in vitro characterize $L$. $(V$.) braziliensis clinical isolates with different responses towards antimony. There is an important heterogeneity among the assayed isolates suggesting that some of them have different innate abilities to adapt to different environments and biological filters. Moreover, serine proteases activity and differential transcripts expression suggested that each isolate may have independent means to adapt to their niches and that in vitro response to antimony needs further characterization, especially in clinical isolates causing ATL. 


\section{Methods}

\section{Chemical, Molecular Biology and Culture Media reagents}

Antibiotics (penicillin and streptomycin), Schneider's insect medium, bovine serum albumin (BSA), Fluorogenic peptide substrate [N-benzyloxycarbonyl---phenylalanyl-I-arginine 7-amino-4-methylcoumarin (Z-FR-AMC)], Proteases inhibitors [trans-epoxysuccinyl--leucylamido(4-guanidino)butane (E-64), Phenylmethylsulfonyl fluoride (PMSF), 4-(2-Aminoethyl)benzenesulfonyl fluoride hydrochloride (AEBSF), Tosyl-L-lysyl-chloromethane hydrochloride (TLCK)], Glucantime ${ }^{\circledR}\left(\mathrm{Sb}^{\vee}\right)$ and antimony potassium tartrate $\left(\mathrm{Sb}^{\mathrm{III}}\right)$ were purchased from Sigma Aldrich Chemical Co. (USA). TRIzol®RNA Isolation Reagent (TRIzol), RNase Henzyme, DEPC-treated water, deoxyribonucleotide phosphate solution (dNTPs), SuperScript III First-strand cDNA Synthesis Kit (SuperScript III kit), Platinum ${ }^{\mathrm{TM}}$ Taq DNA Polymerase (DNA Polymerase), Taq Platinum PCR buffer and AlamarBlue ${ }^{\text {TM }}$ cell viability reagent were purchased from Life Technologies/Thermo Fisher Scientific (USA). Fetal Bovine Serum (FBS) was purchased from Gibco/Thermo Fisher Scientific (Brazil). All reagents were of analytical or superior grade.

\section{Parasite samples}

Twelve L. (V.) braziliensis clinical isolates were acquired from the biological collection of the Evandro Chagas National Institute of Infectious Diseases at Fundação Oswaldo Cruz (INI - Fiocruz). All parasites were isolated before Glucantime ${ }^{\circledR}$ treatment from five patients who were classified as responders $(R)$ since they presented complete lesion healing and clinical cure; and seven non-responders (NR) who had therapeutic failure or relapse. The medical personal of the Leishmaniasis Clinical Research and Vigilance Laboratory (LaPClinVigiLeish) at INI - Fiocruz were responsible for this classification following the criteria reported for ATL patient's treatment in the state of Rio de Janeiro - Brazil $(4,5,54)$. All the isolates included in this study were previously characterized as $L$. $(V$.) braziliensis by multilocus enzyme electrophoresis (MLEE), according to procedures described elsewhere (55). For this study, each isolate was named after its patient' response: Responder (R1, R2, R3, R4, R5) and Non-responder (NR1, NR2, NR3, NR4, NR5, NR6, NR7).

\section{Parasites culture and in vitro differentiation}

Parasites were cultured in biphasic Novy-MacNeal-Nicolle (NNN) medium with $10 \%$ of inactivated FBS. Then, they were expanded in Schneider's insect medium at pH 7.2 supplemented with $20 \%$ of inactivated FBS, $200 \mathrm{IU}$ penicillin and $200 \mathrm{mg} / \mathrm{mL}$ streptomycin and maintained at $26^{\circ} \mathrm{C}$. To obtain each isolate growth curve, $3 \times 10^{5} / \mathrm{mL}$ promastigotes were initially cultured and maintained in $25 \mathrm{~cm}^{2}$ flasks containing $5 \mathrm{~mL}$ of the medium described above. Daily, for eight days, a $10 \mu \mathrm{L}$ aliquot was taken to determine the number of viable parasites using a Neubauer chamber (data not shown). Each parasite isolate did not have more than 7 passages since isolation. Differentiation from promastigotes to axenic amastigotes was performed as previously described elsewhere (38), with a few modifications. Briefly, $5 \times 10^{5}$ per $\mathrm{mL}$ of log-phase promastigotes were cultured in Schneider medium (pH 5.5) supplemented with $20 \%$ of FBS, 60 
IU penicillin, $60 \mathrm{mg} / \mathrm{mL}$ streptomycin and maintained at $26^{\circ} \mathrm{C}$ for 2 days. Then, to complete differentiation, each culture was subjected to temperature shock at $32^{\circ} \mathrm{C}$ for 2 days. After this period, full differentiation was verified under an optical microscope (Labomed, Labo America, Inc.) and these parasites - named as one-day axenic amastigotes - were immediately used for all experimental assays (Supplementary file 8).

\section{In vitro susceptibility assays}

Log-phase promastigotes and one-day axenic amastigotes forms, of each isolate, were tested against $\mathrm{Sb}^{\mathrm{V}}$ and $\mathrm{Sb}^{\mathrm{III}}$ to measure the half-maximal inhibitory concentration $\left(\mathrm{IC}_{50}\right)$ induced by each drug. The $\mathrm{IC}_{50}$ was determined by AlamarBlue ${ }^{\mathrm{TM}}$ reduction assay as previously described (56), with some modifications. Briefly, each parasite form was seeded in 96-well plates in triplicate at adequate conditions: log-phase promastigotes $\left(4 \times 10^{6}\right.$ parasites $\left./ \mathrm{mL}\right)$ and one-day axenic amastigotes $\left(5 \times 10^{5}\right.$ parasites $\left./ \mathrm{mL}\right)$ in $0.1 \mathrm{~mL}$ of Schneider medium (pH 7.2 for promastigotes or $\mathrm{pH} 5.5$ for axenic amastigotes) supplemented with $20 \%$ of FBS and each drug in decreasing concentrations, leaving one column without any drug to serve as the control. $\mathrm{Sb}^{\mathrm{V}}$ concentrations ranged from $20 \mathrm{mg} / \mathrm{mL}$ to $6 \times 10^{-4} \mathrm{mg} / \mathrm{mL}$; Sb ${ }^{111}$ concentrations ranged from $0.196 \mathrm{mg} / \mathrm{mL}$ to $5 \times 10^{-6} \mathrm{mg} / \mathrm{mL}$, with a 2:1 dilution factor between each one. After parasites incubation (Promastigotes: $26^{\circ} \mathrm{C}, 48 \mathrm{~h}$; Axenic amastigotes: $32^{\circ} \mathrm{C}, 24 \mathrm{~h}$ ), AlamarBlue ${ }^{\mathrm{TM}}$ reagent was added to each well $(10 \mu \mathrm{L})$ followed by a new incubation at their respective temperatures for 4 hours. Then, each plate was read on a Spectramax 190 microplate spectrofluorometer (Molecular Devices Corporation) at 570 excitation and $590 \mathrm{~nm}$ emission wavelengths, and the percentage of reduction of AlamarBlue ${ }^{\mathrm{TM}}$ was determined.

\section{Protein extraction}

Log-phase promastigotes $\left(10^{8}\right.$ to $10^{9}$ parasites $\left./ \mathrm{mL}\right)$ and one-day axenic amastigotes $\left(10^{8}\right.$ parasites $\left./ \mathrm{mL}\right)$ of each isolate were separately used to obtain whole protein extracts as it follows. Parasites were washed by centrifugation ( $3000 \times \mathrm{g}, 4^{\circ} \mathrm{C}, 10 \mathrm{~min}$ ) in sterile cold PBS pH 7.2. Then, the pellets were re-suspended in $1 \mathrm{~mL}$ of lysis buffer (100 mM Tris-HCl pH 8.0, $150 \mathrm{mM} \mathrm{NaCl}, 10 \%$ glycerol and $0.6 \%$ Triton X-100) and subjected to a minimum of 5 freeze-thaw cycles. After parasites lysis, confirmed by optical microscopy, the soluble fraction was obtained by centrifugation $\left(25000 \times \mathrm{g}, 4^{\circ} \mathrm{C}, 30 \mathrm{~min}\right)$ and the supernatant stored at $-80^{\circ} \mathrm{C}$ until further use. The parasites total protein concentrations were determined by the Lowry method using BSA as a standard protein (57).

\section{Enzymatic assays}

The serine protease activity of the whole protein extract, $5 \mu \mathrm{g}$ of total protein, was assessed in activation buffer (Tris-HCl [10 mM], pH 7.5) using a specific fluorogenic peptide substrate, Z-FR-AMC [1 mM], at a final volume of $60 \mathrm{~mL}$. Samples were incubated $\left(37^{\circ} \mathrm{C}, 60 \mathrm{~min}\right)$, and the variance in the relative fluorescence was monitored on a Molecular Devices SpectraMax spectrophotometer (Gemini XPS). 
Inhibition assays were performed by incubation $\left(25^{\circ} \mathrm{C}, 5 \mathrm{~min}\right)$ of each sample with specific inhibitors of proteases: E-64 [10 mM] (for cysteine proteases), PMSF [1 mM] (for serine- and cysteine proteases), AEBSF [ $1 \mathrm{mM}$ ] (for serine proteases such as trypsin, chymotrypsin, plasmin, kallikrein and thrombin), and TLCK [100 mM] (for serine proteases such as trypsin and trypsin-like). All inhibitors were assessed at the maximum recommended concentrations (58).

The substrate cleavage rate was defined as follows: $v=D s / D t$, where $v=$ velocity, $D s=$ substrate concentration variation and $\mathrm{Dt}=$ total reaction time, as determined elsewhere (36). The self-degradation of the fluorescent peptide substrate was controlled throughout the assay to avoid incorrect readings; the enzymatic activity is expressed as $\mathrm{mmol} \mathrm{min}^{-1} \cdot \mathrm{mg}$ of protein $^{-1}$.

\section{Primers design}

The primers used in this study were previously designed and used to detect serine proteases and tryparedoxin peroxidase transcripts (26). Briefly, the design is based on the $L$. (V.) braziliensis subtilisins (LbrM.13.0860: sense-5' GAGCTAACACCAGTGGCACA 3' and antisense-5' ATCTGGCGATTTCTCCCTTT 3'; LbrM.28.2570: sense-5' CACTGCGCTCCACATACACT 3' and antisense-5' GCCTTCATTCGAGCTACAGG 3'), tryparedoxin peroxidase (LbrM.15.1080: sense-5' CTCTGTGGACAGCGAGTACG 3' and antisense-5' TGGGGTCGATGATAAAGAGG 3') and 40S Ribosomal Protein S8 housekeeping gene (LbrM.24.2160: sense-5' AGACGCTGGTGAAGAACTGC 3' and antisense-5' AAGTCGATGCCGTAATGCTT 3') sequences recorded in the GeneDB database (http://www.genedb.org). All primers were synthesized by Invitrogen Brazil at a concentration of $50 \mathrm{nM}$ and purified by desalting.

\section{RNA extraction and cDNA synthesis}

Log-phase promastigotes $\left(10^{8}\right.$ to $10^{9}$ parasites $\left./ \mathrm{mL}\right)$ and one-day axenic amastigotes $\left(10^{7}\right.$ parasites $\left./ \mathrm{mL}\right)$ were separately lysed in $1 \mathrm{~mL}$ TRIzol containing $200 \mathrm{~mL}$ of chloroform. For RNA extraction the samples were centrifuged $\left(10000 \times \mathrm{g}, 4^{\circ} \mathrm{C}, 10 \mathrm{~min}\right)$ and the supernatants containing RNA were dissolved in Isopropanol $\left(12000 \times \mathrm{g}, 4^{\circ} \mathrm{C}, 20 \mathrm{~min}\right)$ and washed with $70 \%$ ethanol $\left(9000 \times \mathrm{g}, 4^{\circ} \mathrm{C}, 5 \mathrm{~min}\right)$. Then, each pellet was re-suspended in 30 to $40 \mathrm{~mL}$ of DEPC-treated water and incubated $\left(56^{\circ} \mathrm{C}, 10 \mathrm{~min}\right)$ to complete dissolution. The RNA concentrations were measured by spectrophotometry at 260/280 nm and 230/260 $\mathrm{nm}$. DNAse treatment and cDNA synthesis were performed using the SuperScript ${ }^{\circledR}$ III Kit with a maximum of $4 \mu \mathrm{g}$ of total RNA. cDNA concentration of each sample was measured with Qubit ${ }^{\mathrm{TM}}$ ssDNA Assay Kit (Thermo Fisher Scientific), following the manufacturer's protocol.

\section{Reverse Transcription Polymerase Chain Reaction (RT-PCR)}

Primers complementary to the LbrM.13.0860, LbrM.28.2570, LbrM.15.1080 and LbrM.24.2160 genes were used for conventional PCR under the following reaction conditions: $10 X$ PCR Buffer, $50 \mathrm{mM} \mathrm{MgCl}$, $10 \mathrm{mM}$ of each dNTP, $0.3 \mu \mathrm{M}$ of each primer and $1 \mathrm{U}^{\text {Platinum }}{ }^{\mathrm{TM}}$ Taq DNA polymerase, together with $20 \mathrm{ng}$ of cDNA, adjusted to a final volume of $25 \mu \mathrm{L}$. The amplification cycles were: initial denaturation $\left(94^{\circ} \mathrm{C}, 3\right.$ min), 40 cycles of denaturation, hybridization and extension $\left(95^{\circ} \mathrm{C}, 20 \mathrm{~s} ; 56^{\circ} \mathrm{C}, 30 \mathrm{~s} ; 72{ }^{\circ} \mathrm{C}, 30 \mathrm{~s}\right)$ and a 
final extension step $\left(72{ }^{\circ} \mathrm{C}, 5 \mathrm{~min}\right)$ using a PTC-100 Thermal Cycler (Applied Biosystems, Foster City, CA, USA). The amplified products were evaluated by $1.2 \%(\mathrm{w} / \mathrm{v})$ agarose gel electrophoresis and stained with ethidium bromide.

\section{Statistical analysis}

GraphPad Prism software (version 8.3.0) was used to calculate $I_{50}$ values and establish significant differences by Student's T-test, considering $p<0.05$ as a significant difference. While, to compare inhibition regimens (W/i, E-64, PMSF, AEBSF, TLCK) in each isolate, we used 2way ANOVA followed by Dunnett's multiple comparison test.

Additionally, the quantitative variables obtained for each clinical isolate: i) promastigotes $\mathrm{Sb}^{\vee} \mathrm{IC}_{50}$, (ii)

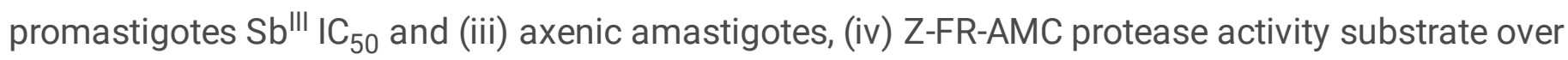
promastigotes and (v) axenic amastigotes, (vi) PMSF inhibition over promastigotes and (vii) axenic amastigotes, (viii) AEBSF inhibition over promastigotes and (ix) axenic amastigotes, (x) TLCK inhibition over promastigotes and (xi) axenic amastigotes were normalized and subjected to principal component analysis (PCA). Here we have 11 variables per isolate and biological form which is why PCA analysis helped us reduce dimensionality and perform further cluster analysis (59). The first three PCs (PC1, PC2, PC3) were used to cluster the clinical isolates using the K-means algorithm clustering method. To determine the optimal number of clusters we used the total-within cluster sum of squares (twcss), as a function of the number of clusters, where the squared distances between each cluster centroid 0 and each of its cluster members ( are summed over each cluster, Nc is the total number of clusters, equation (1). Additionally, with the previously normalized data, we explored the characteristics of the clusters by calculating the pairwise distance between clusters using the normalized values of $\mathrm{Sb}^{\mathrm{II}} \mathrm{IC}_{50}$, PMSF, AEBSF and TLCK inhibition over promastigotes and axenic amastigotes. The statistical analysis was carried out using R (version 1.1.463).

$$
\text { WCSS }=\sum_{i=1}^{N c} \sum_{x \in c_{i}}^{N} d\left(x, \bar{x}_{c_{i}}\right)^{2}
$$

\section{References}

1. WHO. Leishmaniasis [Internet]. 2019 [cited 2019 Nov 26]. Available from: https://www.who.int/newsroom/fact-sheets/detail/leishmaniasis

2. Akhoundi M, Kuhls K, Cannet A, Votýpka J, Marty P, Delaunay P, et al. A Historical Overview of the Classification, Evolution, and Dispersion of Leishmania Parasites and Sandflies. PLOS Neglected Tropical Diseases. 2016 Mar 3;10(3):e0004349.

3. Lima EB de, Porto C, Motta JOC da, Sampaio RNR. Tratamento da Leishmaniose Tegumentar Americana. Anais Brasileiros de Dermatologia. 2007 Apr;82(2):111-24. 
4. Saheki MN, Lyra MR, Bedoya-Pacheco SJ, Antônio L de F, Pimentel MIF, Salgueiro M de M, et al. Low versus high dose of antimony for American cutaneous leishmaniasis: A randomized controlled blind non-inferiority trial in Rio de Janeiro, Brazil. PLoS One [Internet]. 2017 May 30 [cited 2020 Jun 16];12(5). Available from: https://www.ncbi.nlm.nih.gov/pmc/articles/PMC5448803/

5. Cataldo Jl, Conceição-Silva F, Antônio L de F, Schubach A de O, Marzochi MC de A, Valete-Rosalino $\mathrm{CM}$, et al. Favorable responses to treatment with $5 \mathrm{mg} \mathrm{Sbv} / \mathrm{kg} /$ day meglumine antimoniate in patients with American tegumentary leishmaniasis acquired in different Brazilian regions. Rev Soc Bras Med Trop. 2018 Dec;51(6):769-80.

6. Frézard F, Demicheli C, Ferreira CS, Costa MAP. Glutathione-Induced Conversion of Pentavalent Antimony to Trivalent Antimony in Meglumine Antimoniate. Antimicrob Agents Chemother. 2001 Mar;45(3):913-6.

7. Frézard F, Demicheli C, Kato KC, Reis PG, Lizarazo-Jaimes EH. Chemistry of antimony-based drugs in biological systems and studies of their mechanism of action. Reviews in Inorganic Chemistry. 2013 May 1;33(1):1-12.

8. Wyllie S, Cunningham ML, Fairlamb AH. Dual Action of Antimonial Drugs on Thiol Redox Metabolism in the Human Pathogen Leishmania donovani. J Biol Chem. 2004 Sep 17;279(38):39925-32.

9. Flohé L, Steinert P, Hecht H-J, Hofmann B. Tryparedoxin and tryparedoxin peroxidase. Meth Enzymol. 2002;347:244-58.

10. Frézard F, Monte-Neto R, Reis PG. Antimony transport mechanisms in resistant leishmania parasites. Biophys Rev. 2014 Jan 25;6(1):119-32.

11. Singh N. Drug resistance mechanisms in clinical isolates of Leishmania donovani. Indian J Med Res. 2006 Mar;123(3):411-22.

12. Ponte-Sucre A, Gamarro F, Dujardin J-C, Barrett MP, López-Vélez R, García-Hernández R, et al. Drug resistance and treatment failure in leishmaniasis: A 21st century challenge. PLoS Negl Trop Dis. 2017 Dec;11(12):e0006052.

13. Baptista C, Miranda L de FC, Madeira M de F, Leon LLP, Conceição-Silva F, Schubach A de O. In Vitro Sensitivity of Paired Leishmania (Viannia) braziliensis Samples Isolated before Meglumine Antimoniate Treatment and after Treatment Failure or Reactivation of Cutaneous Leishmaniasis. Dis Markers [Internet]. 2015 [cited 2020 Aug 24];2015. Available from: https://www.ncbi.nlm.nih.gov/pmc/articles/PMC4329789/

14. Croft SL, Sundar S, Fairlamb AH. Drug Resistance in Leishmaniasis. Clinical Microbiology Reviews. 2006 Jan 1;19(1):111-26.

15. Jeddi F, Piarroux R, Mary C. Antimony Resistance in Leishmania, Focusing on Experimental Research [Internet]. Journal of Tropical Medicine. 2011 [cited 2020 Mar 3]. Available from: https://www.hindawi.com/journals/jtm/2011/695382/

16. Denton H, McGREGOR JC, Coombs GH. Reduction of anti-leishmanial pentavalent antimonial drugs by a parasite-specific thiol-dependent reductase, TDR1. Biochemical Journal. $2004 \mathrm{Jul}$ 15;381(2):405-12. 
17. Zhou Y, Messier N, Ouellette M, Rosen BP, Mukhopadhyay R. Leishmania majorLmACR2 Is a Pentavalent Antimony Reductase That Confers Sensitivity to the Drug Pentostam. J Biol Chem. 2004 Sep 3;279(36):37445-51.

18. Alves CR, Souza RS de, Charret K dos S, Côrtes LM de C, Sá-Silva MP de, Barral-Veloso L, et al. Understanding serine proteases implications on Leishmania spp lifecycle. Experimental Parasitology. 2018 Jan;184:67-81.

19. Borsari C, Quotadamo A, Ferrari S, Venturelli A, Cordeiro-da-Silva A, Santarem N, et al. Chapter Two Scaffolds and Biological Targets Avenue to Fight Against Drug Resistance in Leishmaniasis. In: Botta M, editor. Annual Reports in Medicinal Chemistry [Internet]. Academic Press; 2018 [cited 2019 Nov 27]. p. 39-95. (Neglected Diseases: Extensive Space for Modern Drug Discovery; vol. 51). Available from: http://www.sciencedirect.com/science/article/pii/S0065774318300022

20. da Silva-Lopez RE, Giovanni-De-Simone S. Leishmania (Leishmania) amazonensis: purification and characterization of a promastigote serine protease. Experimental Parasitology. 2004 Jul 1;107(3):173-82.

21. Guedes HLM, Rezende NJM, Fonseca MA, Salles CMC, Rossi-Bergmann B, De-Simone G. Identification of Serine Proteases from Leishmania braziliensis. Zeitschrift für Naturforschung C. 2014;62(5-6):373-381.

22. Machado P de A, Carneiro MPD, Sousa-Batista A de J, Lopes FJP, Lima APC de A, Chaves SP, et al. Leishmanicidal therapy targeted to parasite proteases. Life Sciences. 2019 Feb 15;219:163-81.

23. Pereira B, Souza-Silva F, Silva-Almeida M, Santos-de-Souza R, Oliveira L, Ribeiro-Guimaraes M, et al. Proteinase Inhibitors: A Promising Drug Class for Treating Leishmaniasis. Current Drug Targets. 2014 Nov 7;15(12):1121-31.

24. Silva-López RE, Santos TR dos, Morgado-Díaz JA, Tanaka MN, De Simone SG. Serine protease activities in Leishmania (Leishmania) chagasi promastigotes. 2010 [cited 2020 Mar 3]; Available from: https://www.arca.fiocruz.br/handle/icict/21057

25. Swenerton RK, Knudsen GM, Sajid M, Kelly BL, McKerrow JH. Leishmania Subtilisin Is a Maturase for the Trypanothione Reductase System and Contributes to Disease Pathology. J Biol Chem. 2010 Oct 8;285(41):31120-9.

26. Santos-de-Souza R, Monteiro de Castro Côrtes L, dos Santos Charret K, Cysne-Finkelstein L, Alves CR, Souza-Silva F. Serine Proteinases in Leishmania (Viannia) braziliensis Promastigotes Have Distinct Subcellular Distributions and Expression. International Journal of Molecular Sciences. 2019;20(6):1315.

27. Rodrigues AM, Hueb M, Santos TARR dos, Fontes CJF. Fatores associados ao insucesso do tratamento da leishmaniose cutânea com antimoniato de meglumina. Revista da Sociedade Brasileira de Medicina Tropical. 2006 Apr;39(2):139-45.

28. Rojas R, Valderrama L, Valderrama M, Varona MX, Ouellette M, Saravia NG. Resistance to Antimony and Treatment Failure in Human Leishmania (Viannia) Infection. J Infect Dis. 2006 May $15 ; 193(10): 1375-83$. 
29. Soto J, Soto P. Current situation and future of antileihmanial therapy in Colombia. Biomédica. 2006 Oct 1;26:194-206.

30. Heitman J. Sexual Reproduction and the Evolution of Microbial Pathogens. Current Biology. 2006 Sep;16(17):R711-25.

31. Azeredo-Coutinho RBG, Mendonça SCF, Callahan H, Portal AC, Grögl M. Sensitivity of leishmania braziliensis promastigotes to meglumine antimoniate (glucantime) is higher than that of other leishmania species and correlates with response to therapy in american tegumentary leishmaniasis. Journal of Parasitology. 2007 Jun 1;93(3):688-93.

32. Gagini T, de Oliveira Schubach A, de Fatima Madeira M, Maria Valete-Rosalino C, Fernandes Pimentel MI, da Silva Pacheco R. Genotypic profiles of Leishmania (Viannia) braziliensis strains from cutaneous leishmaniasis patients and their relationship with the response to meglumine antimoniate treatment: a pilot study. Parasite [Internet]. [cited 2020 Jun 15];24. Available from: https://www.ncbi.nlm.nih.gov/pmc/articles/PMC5621350/

33. Ephros M, Bitnun A, Shaked P, Waldman E, Zilberstein D. Stage-Specific Activity of Pentavalent Antimony against Leishmania donovani Axenic Amastigotes. Antimicrob Agents Chemother. 1999 Feb;43(2):278-82.

34. Sereno D, Cavaleyra M, Zemzoumi K, Maquaire S, Ouaissi A, Lemesre JL. Axenically Grown Amastigotes of Leishmania infantum Used as an In Vitro Model To Investigate the Pentavalent Antimony Mode of Action. Antimicrob Agents Chemother. 1998 Dec;42(12):3097-102.

35. Aït-Oudhia K, Gazanion E, Vergnes B, Oury B, Sereno D. Leishmania antimony resistance: what we know what we can learn from the field. Parasitol Res. 2011 Nov;109(5):1225-32.

36. Alves CR, Corte-Real S, Bourguignon SC, Chaves CS, Saraiva EMB. Leishmania amazonensis: early proteinase activities during promastigote-amastigote differentiation in vitro. Experimental Parasitology. 2005 Jan 1;109(1):38-48.

37. Cysne-Finkelstein L, Silva-Almeida M, Pereira BAS, dos Santos Charret K, Bertho ÁL, Bastos LS, et al. Evidence of Subpopulations with Distinct Biological Features Within a Leishmania (Viannia) braziliensis Strain. Protist. 2018 Feb 1;169(1):107-21.

38. Gomes CB, -Silva FS, Charret KDS, Pereira BAS, Finkelstein LC, Santos-de-Souza R, et al. Increasing in cysteine proteinase $B$ expression and enzymatic activity during in vitro differentiation of Leishmania (Viannia) braziliensis: First evidence of modulation during morphological transition. Biochimie. 2017 Feb;133:28-36.

39. Nasereddin A, Schweynoch C, Schonian G, Jaffe CL. Characterization of Leishmania (Leishmania) tropica axenic amastigotes. Acta Tropica. 2010 Jan 1;113(1):72-9.

40. Saunders EC, Ng WW, Kloehn J, Chambers JM, Ng M, McConville MJ. Induction of a Stringent Metabolic Response in Intracellular Stages of Leishmania mexicana Leads to Increased Dependence on Mitochondrial Metabolism. PLoS Pathog [Internet]. 2014 Jan 23 [cited 2020 Aug 5];10(1). Available from: https://www.ncbi.nlm.nih.gov/pmc/articles/PMC3900632/ 
41. Morita T, Kato H, Iwanaga S, Takada K, Kimura T, Sakakibara S. New Fluorogenic Substrates for aThrombin, Factor Xa, Kallikreins, and Urokinase. The Journal of Biochemistry. 1977;82(5):1495-8.

42. Basak S, Stewart NA, Chrétien M, Basak A. Aminoethyl benzenesulfonyl fluoride and its hexapeptide (Ac-VFRSLK) conjugate are both in vitro inhibitors of subtilisin kexin isozyme-1. FEBS Letters. 2004 Aug 27;573(1):186-94.

43. Silva CL. Caracterização bioquímica da Prolil Oligopeptidase de Leishmania chagasi, um potencial alvo quimioterápico para as Leishmanioses. 2014 Jan 23 [cited 2020 Mar 6]; Available from: https://repositorio.unb.br/handle/10482/16720

44. Wang R, Liu S, Wang J, Dong Q, Xu L, Rui Q. Purification, characterization and identification of a senescence related serine protease in dark-induced senescent wheat leaves. Phytochemistry. 2013 Nov 1;95:118-26.

45. Cupolillo E, Brahim LR, Toaldo CB, Oliveira-Neto MP de, Brito MEF de, Falqueto A, et al. Genetic Polymorphism and Molecular Epidemiology of Leishmania (Viannia) braziliensis from Different Hosts and Geographic Areas in Brazil. Journal of Clinical Microbiology. 2003 Jul 1;41(7):3126-32.

46. Schriefer A, Schriefer ALF, Góes-Neto A, Guimarães LH, Carvalho LP, Almeida RP, et al. Multiclonal Leishmania braziliensis Population Structure and Its Clinical Implication in a Region of Endemicity for American Tegumentary Leishmaniasis. Infection and Immunity. 2004 Jan 1;72(1):508-14.

47. Chu N-M, Chao Y, Bi R-C. The 2 A crystal structure of subtilisin E with PMSF inhibitor. Protein Eng Des Sel. 1995 Mar 1;8(3):211-5.

48. Moreira D de S, Pescher P, Laurent C, Lenormand P, Späth GF, Murta SMF. Phosphoproteomic analysis of wild-type and antimony-resistant Leishmania braziliensis lines by 2D-DIGE technology. PROTEOMICS. 2015;15(17):2999-3019.

49. Wyllie S, Mandal G, Singh N, Sundar S, Fairlamb AH, Chatterjee M. Elevated levels of tryparedoxin peroxidase in antimony unresponsive Leishmania donovani field isolates. Molecular and Biochemical Parasitology. 2010 Oct 1;173(2):162-4.

50. Kumar D, Singh R, Bhandari V, Kulshrestha A, Negi NS, Salotra P. Biomarkers of antimony resistance: need for expression analysis of multiple genes to distinguish resistance phenotype in clinical isolates of Leishmania donovani. Parasitology Research. 2012 Jul;111(1):223-30.

51. Oliaee RT, Sharifi I, Afgar A, Kareshk AT, Asadi A, Heshmatkhah A, et al. Unresponsiveness to meglumine antimoniate in anthroponotic cutaneous leishmaniasis field isolates: analysis of resistance biomarkers by gene expression profiling. Trop Med Int Health. 2018 Apr 30;

52. Rugani JN, Gontijo CMF, Frézard F, Soares RP, do Monte-Neto RL. Antimony resistance in Leishmania (Viannia) braziliensis clinical isolates from atypical lesions associates with increased ARM56/ARM58 transcripts and reduced drug uptake. Mem Inst Oswaldo Cruz [Internet]. 2019 Aug 15 [cited 2020 Feb 20];114. Available from: https://www.ncbi.nlm.nih.gov/pmc/articles/PMC6697410/

53. Gómez MA, Olivier M. Proteases and phosphatases during leishmania-macrophage interaction: Paving the road for pathogenesis. Virulence. 2010 Jul;1(4):314-8. 
54. Brahim LR, Valete-Rosalino CM, Antônio L de F, Pimentel MIF, Lyra MR, Paes LE de C, et al. Low dose systemic or intralesional meglumine antimoniate treatment for American tegumentary leishmaniasis results in low lethality, low incidence of relapse, and low late mucosal involvement in a referral centre in Rio de Janeiro, Brazil (2001-2013). Memórias do Instituto Oswaldo Cruz. 2017 Dec;112(12):83843.

55. Cupolillo E, Jr GG, Momen H. A General Classification of New World Leishmania Using Numerical Zymotaxonomy. The American Journal of Tropical Medicine and Hygiene. 1994 Mar 1;50(3):296311.

56. Shimony O, Jaffe CL. Rapid fluorescent assay for screening drugs on Leishmania amastigotes. J Microbiol Methods. 2008 Oct;75(2):196-200.

57. Lowry OH, Rosebrough NJ, Farr AL, Randall RJ. Protein measurement with the Folin phenol reagent. Journal of Biological Chemistry. 1951;193:265-75.

58. Beynon RJ, Bond JS, editors. Proteolytic enzymes: a practical approach. Oxford; New York: IRL Press at Oxford University Press; 1989. 259 p. (The Practical approach series).

59. Bartholomew DJ. Principal Components Analysis. In: Peterson P, Baker E, McGaw B, editors. International Encyclopedia of Education (Third Edition) [Internet]. Oxford: Elsevier; 2010 [cited 2020 Dec 14]. p. 374-7. Available from:

http://www.sciencedirect.com/science/article/pii/B9780080448947013580

\section{Declarations}

\section{Acknowledgements}

We are grateful to the technical support of the Instituto Oswaldo Cruz platforms: Bioassays and Drug Screening, Culture Medium and Water Platform Grade Reagent Type I and II. Conselho Nacional de Desenvolvimento Científico e Tecnológico - Brasil (CNPq) and Coordenação de Aperfeiçoamento de Pessoal de Nível Superior - Brasil (CAPES).

\section{Author's contribution}

AZP conducted all the experiments, data analysis and wrote the original manuscript. GDL, LCF, FSS helped in the experiments planning and execution. FCS, LFCM provided the frozen parasite isolates. AF, MIFP, AOS provided information about the clinical origin of the isolates. AZP, LAM conducted the statistical analysis. GDL, CRA critically reviewed the data analysis and final manuscript.

\section{Competing interests}

The authors declare no competing interests.

\section{Funding}

This study was financed in part by the CNPq (301744/2019-0) and CAPES (Finance Code 001). 


\section{Figures}
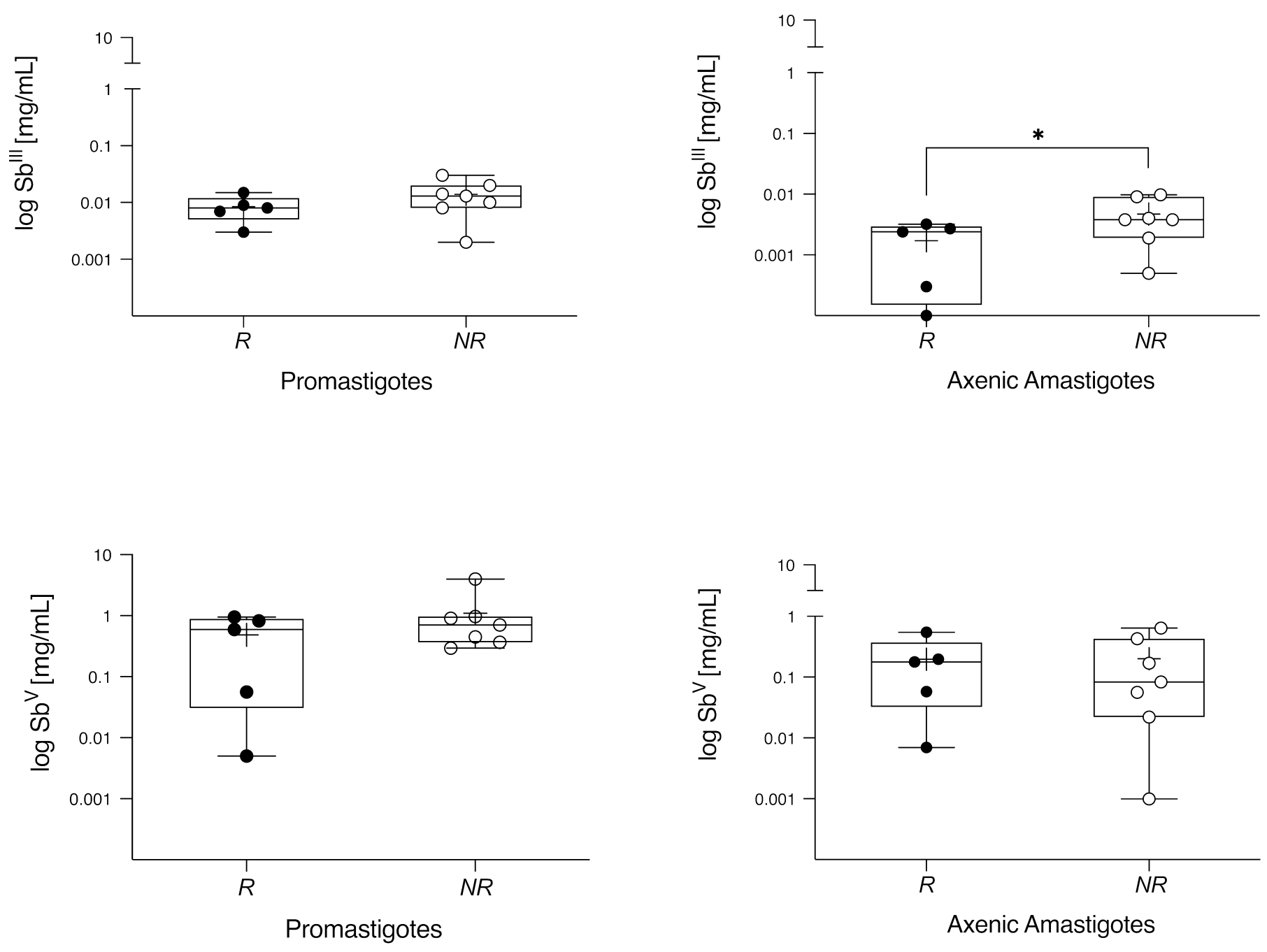

\section{Figure 1}

Promastigotes and axenic amastigotes in vitro susceptibility profile to antimony. Both parasite forms of each isolate $(n=12), 4 \times 106$ promastigotes/well and $5 \times 105$ axenic amastigotes/well were exposed to serial dilutions of trivalent (SbIII) and pentavalent (SbV) antimonial for 48 hours and 24 hours in 96-well plates, respectively. The half-maximal inhibitory concentration (IC50 $[\mathrm{mg} / \mathrm{mL}])$ of parasites viability was measured using a fluorescence method with AlamarBlue reagent. Parasites isolated before treatment of patients with ATL cured after antimonial therapy (R: $\bullet$ ) or with poor clinical response to therapy, either therapeutic failure or relapse (NR: $\bullet$ ). The data is presented by boxplot diagrams as the mean of three biological replicates for each isolate. Asterisks indicate statistically significant differences: * $p<0.05$ 


\section{Promastigotes}

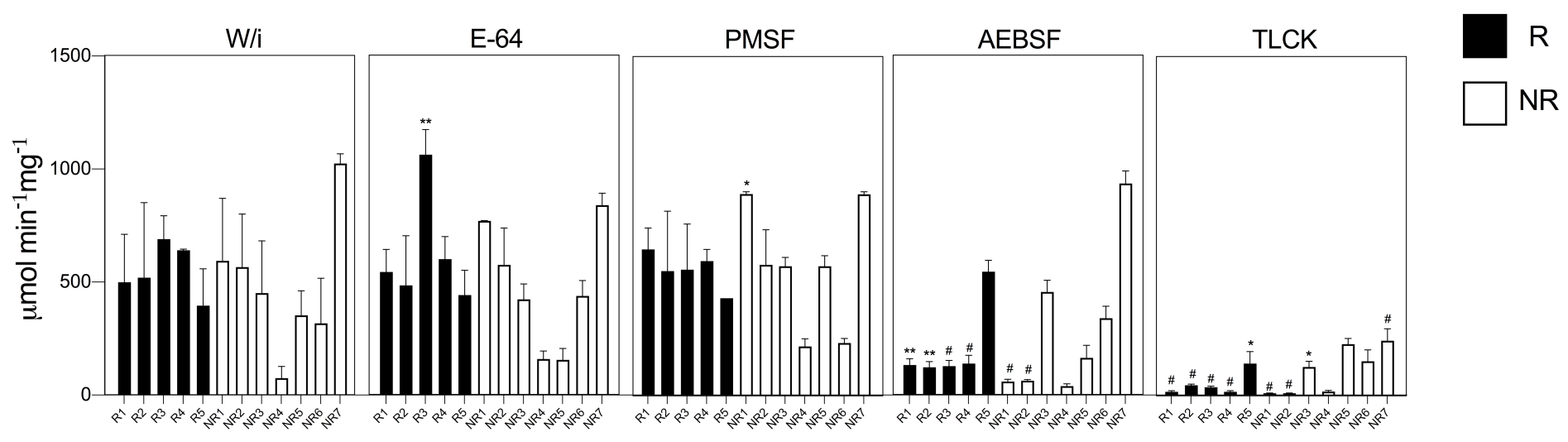

Axenic Amastigotes

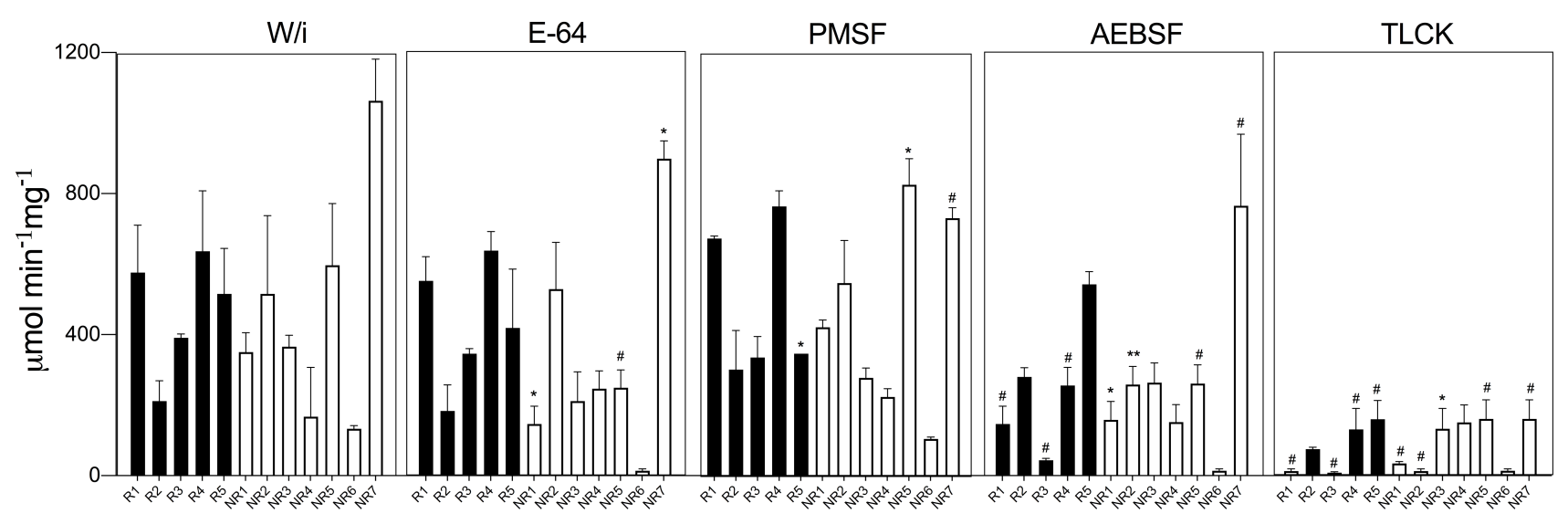

Figure 2

Enzymatic residual activity of whole soluble proteins from the clinical isolates. In these assays, protein extract [5 $\mathrm{\mu g}$ ] of promastigotes and axenic amastigotes were measured using a specific fluorogenic peptide substrate of serine proteinases, z-FR-AMC $(1 \mathrm{mM})$. The enzymatic activities ( $\mathrm{mmol}$ min-1.mg of protein-1) were assessed without inhibitor (w/i) and in the presence of inhibitors: E-64 [10 $\mu \mathrm{M}]$, PMSF [1 $\mathrm{mM}]$, AEBSF [ $1 \mathrm{mM}]$ and TLCK [100 $\mu \mathrm{M}]$. R: Responder; NR: Non-responder. The results are shown as mean and the standard deviation ( \pm ) of three independent experiments. Asterisks indicate statistically significant differences of enzymatic activities in the absence (w/i) and presence (E-64, PMSF, AEBSF,

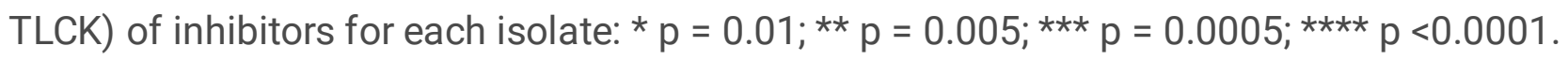




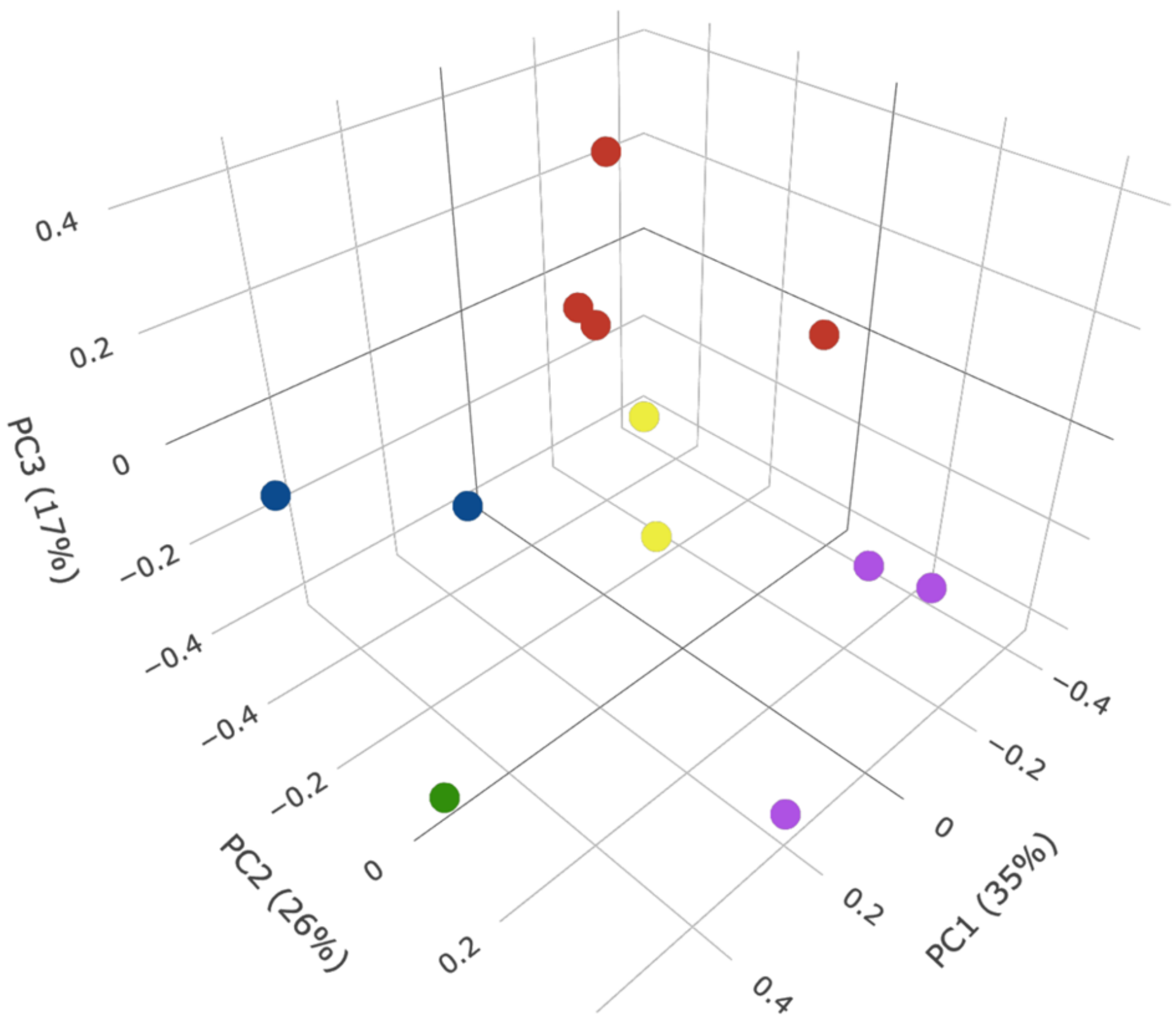

Figure 3

Cluster analysis of L. (V.) braziliensis clinical isolates. Principal components analysis (PCA) was performed to group clinical isolates based on the normalized quantitative variables. Each point represents the first three principal components of a clinical isolate. The points are coloured according to the cluster they belong to, C1 (blue): NR6 and R3; C2 (red): R1, R3, NR5 and NR7; C3 (green): NR3; C4 (purple): NR2, R2 and R4; C5 (yellow): NR1 and NR4. 

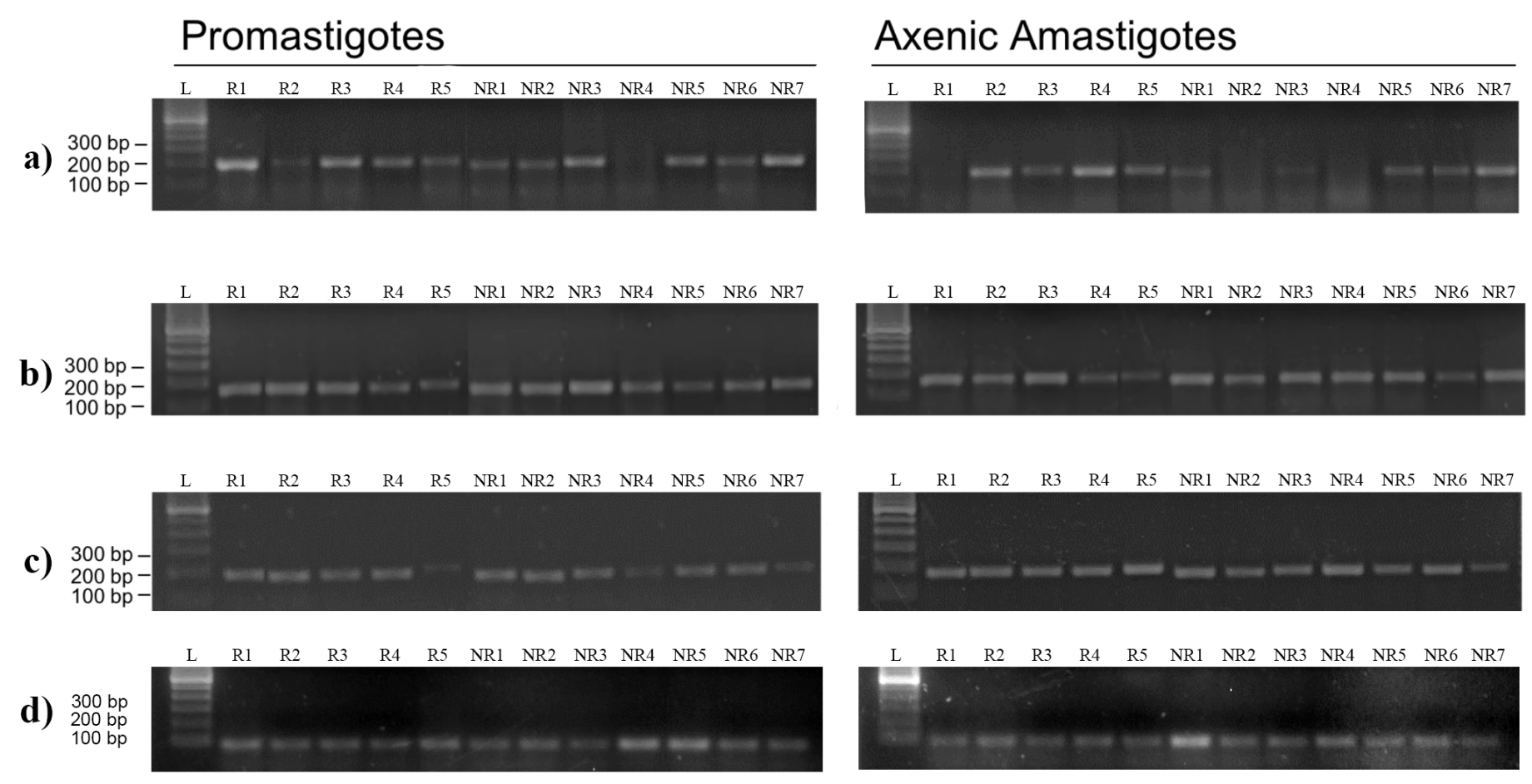

\section{Figure 4}

Detection of RNA transcripts in the isolates. The RT-PCR assays were performed using specific primers sets: a) LbrM.13.0860, subtilisin gene (200 bp); b) LbrM.28.2570, subtilisin gene (162 bp); c) LbrM.15.1080, tryparedoxin peroxidase gene (166 bp); and d) LbrM.24.2160, 40S Ribosomal Protein S8 the housekeeping gene used (98 bp). The amplified products of clinical isolates (1 to 12) were assessed by agarose gels electrophoresis ( $2 \%$ agarose). Lanes (L) are 100 bp DNA ladder (Invitrogen), used as a molecular weight marker.

\section{Supplementary Files}

This is a list of supplementary files associated with this preprint. Click to download.

- SupplementaryfilesSR.docx 